\title{
Synergistic Interaction of Natural Snail Mucin and Lincomycin for Immuno-chemotherapy against Streptopneumococcal Infection: Checkerboard Evaluations
}

\author{
Thaddeus Harrison Gugu ${ }^{1,2, *}$, Restus Chibuike Onwusoba ${ }^{1}$, Patrick Ndubuisi Onyi ${ }^{1}$, Angela Chizoba Ozioko ${ }^{1}$ \\ 'Department of Pharmaceutical Microbiology and Biotechnology, University of Nigeria, NsuKKA Enugu, NIGERIA. \\ 2Department of Pharmaceutics, SNJB's Shriman Sureshdada Jain College of Pharmacy, Chandwad, University of Pune, Savitribai Phule Pune University, Pune, \\ Maharashtra, INDIA.
}

\begin{abstract}
Introduction: Pneumonia is an acute infection of lung majorly caused by Streptoneumococcal bacteria or other microbes which present cough with phlegm (mucus), fever and other clinical symptoms. This mucus produced are easily polymerize with other similar natural product like snail mucin when taken which serves as a natural antimicrobial immune booster for enhanced or synergistic response against causative agent in the presence of antibiotics. This work was carried out to determine the combined effect of snail mucin as animal sourced natural product antimicrobial agent with lincomycin against different strains of Streptococcus pneumoniae as alternative treatment option for this respiratory tract infection "pneumonia" which has been a global challenge especially in the tropical Africa. Methods: Separate solution each of the two agent was prepared in sterile normal saline to get a different concentration of the agents, each solution contained twice the MIC of the agent. The agents were then combined in different ratios for interaction effect by adopting the standard continuous variation method and further diluted using two-fold serially dilutions and evaluated against the test organisms using both agar diffusion and dilution method respectively. Results: The result of FIC index was calculated
\end{abstract}

showed greater synergy in the equal ratio $(50: 50)$ combination against all the strains of tested organisms. Also, better performance at the combinations ratios of $60: 40$ and 40:60 of mucin and lincomycin respectively emerged. Conclusion: This synergistic effect has shown an improved natural product immuno-antimicrobial chemotherapy for alternative treatment option of such acute infection in the absent orthodox medicine.

Key words: Mucin, Lincomycin, Pneumonia, Streptococcus pneumoniae, Synergistic.

\section{Correspondence}

Dr. Thaddeus H Gugu

'Department of Pharmaceutical Microbiology and biotechnology, University of $\mathrm{Ni}-$ geria, NsuKKA Enugu, NIGERIA.

2Department of Pharmaceutics, SNJB's Shriman Sureshdada Jain College of Pharmacy, Chandwad, University of Pune, Savitribai Phule Pune University, Pune, Maharashtra, INDIA.

Phone no: +2348034152833

Email: thaddeus.gugu@unn.edu.ng

DOI: 10.5330/ijpi.2020.3.67

\section{INTRODUCTION}

Pneumonia infection is a diseases of the lower respiratory tract which suffers the invasion of an infective micro-organism and can be community or nosocomial infectious acquired in the hospital setting respiration or inhalation of the pathogenic organism. ${ }^{1}$ Occasionally, inhaled chemicals that irritate the lungs can also cause pneumonia and this can obstruct oxygen requirement or its circulation for normal respiration which result to painful and difficult respiration and can lead to lung injury. ${ }^{2}$ With the advent of antibiotics, the threat from such ailment was able to reduce but recently, Community-acquired pneumonia caused by some Gramnegative bacteria has recorded related multidrug antibiotic resistance to some drugs used in treatment of such diseases. ${ }^{3}$ This situation has led to resort on herbal or natural products of both plants and animals origin. The prevalence of bacterial resistance to antibiotics strengthen the need to ascertain the potency of antibiotics against infectious agents. ${ }^{4}$ Use of drug-drug and drug with other natural product combinations to enhance the activities of the combined antibiotics synergistically has become paramount to combat resistance. ${ }^{5}$ A common feature of mucin as mucus constitutes an essential feature of the innate immune system which exist across both aquatic and terrestrial metazoans. ${ }^{6}$ This plays a vital role in the prevention of disease upon exposure to the environmental microbes and other potential pathogens. ${ }^{7}$ Mucin, apart from a high molecular weight and a high carbohydrate content
(68-81\%), is the abundance of negatively charged groups. The negative charges arise mainly from sialic acid residues and in some cases from sulphated sugars. These acidic groups account for the low isoelectric point (2-3) of mucins. ${ }^{89}$ The basic amino acids of the protein component are serine and threonine. The glycosylated regions of mucins interact favorably with water and force the molecule to an extended random coil conformation and the high molecular weight enables individual mucin molecules to overlap and entangle at relatively low concentrations. ${ }^{10}$

Snail of Achatina fulica is widely use as both meat in some local setting as source of protein and iron with low fats. ${ }^{11,12}$ Also, most of animal feed contain the snail grinded shell as its component for minerals such as calcium for bone and egg shell formation, ${ }^{13}$ as well as other minerals like potassium and magnesium. In cosmetics industry, snail mucus (mucin) organic compound like allantoin, hyaluronic acid are used in production of skin care and peptide antibiotics for pathogens of bacteria. ${ }^{14}$

Lincomycin is an antibiotic of lincosamide obtained from fermentation product of Streptomyces lincolnensis that has been hydrated as lincomycin hydrochloride as a white crystalline powder which has odorless or faint odor with bitter taste. ${ }^{15}$ It is a narrow spectrum antibiotic in form of lincomycin hydrochloride with activity against Gram-positive and cell wall-less bacteria including pathogenic species of Streptococcus, Staphylococcus and Mycoplasma. 

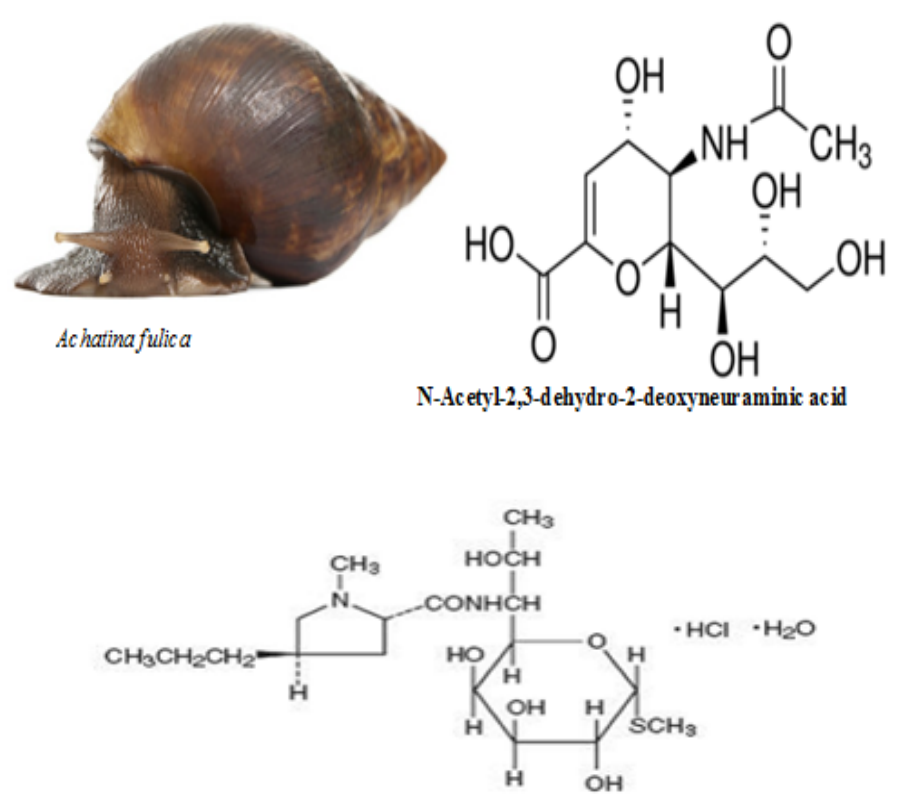

Lincomycin hydrochloride

Lincomycin hydrochloride is freely soluble in water, soluble in dimethylformamide and very slightly soluble in acetone. It has some side effect of diarrhea, chills, fever. ${ }^{16}$

In the treatment of an infection or disease with combined chemotherapeutic agent, the use of an antimicrobial combination can only be encouraged and justifiable if the combinations produce synergistic effect against the causative agents or the organisms. ${ }^{17}$ The use of animal product like snail slime as source of antibacterial agent has proven a better effect when combined with some natural or synthetic chemicals. ${ }^{18}$ Although synergism between some antimicrobial may not occur in some cases. Strategies to combat resistance has been recently developed through drug-drug in combination for effective therapeutic output. ${ }^{5}$ Decades ago, clinical effort has directed at identifying drug combinations that most effectively kill the widest range of pathogens. ${ }^{19,20}$ Drugs and other natural product combinations have been widely researched on and identified more interesting activities focusing mainly on improving the activity of individual drug synergistically through chemical interaction. ${ }^{21}$ The checkerboard assay techniques involves the use of microbiological method to evaluate combined effect of two or more different antimicrobial agents. There are two variations of the checkerboard method which include the fractional inhibitory concentration (FIC) Index and Isologram..$^{22}$ The fractional inhibitory concentration of an agent is the minimum inhibitory concentration in the combination divided by the independent MIC of the agent as in case may be. The sum of the FICs of both agents can be regarded as the FIC Index. This can be represented as

FIC Index $=\frac{A^{\prime}}{A^{\prime \prime}}+\frac{B^{\prime}}{B^{\prime \prime}}$

Where $\mathrm{A}^{\prime}$ and $\mathrm{B}$ is the minimum concentration of agent $\mathrm{A}$ and $\mathrm{B}$ having inhibitory effects when acting together also called individual MIC in combination, while $\mathrm{A}^{\prime \prime}$ and $\mathrm{B}$ " is the respective MICs of the agents and this can be regarded as individual effect without combination.

\section{Materials and methods}

Weighing Balance (Scout pro u401 made in China), binocular microscope (Olympus)glassware's, incubator (GenLab, UK), hot air oven (GenLab, UK), autoclave (Gallynkamp England), stainless bowel and tray, test tube (pyrex) racks,glas slide, syringes and needle, Micropipette, glass rod, inoculation loop, bunsen burner, MacCartney bottle, sample bottles, swab sticks, cotton wool.

The media and reagents used include: Coloumbia blood agar base (Oxoid Ltd UK), Mueller-Hinton agar (Oxoid Ltd UK), bile salt, hydrogen peroxide, normal saline, Lincomycin (Pauco Pharmaceutical Industries Nigeria Limited Abakaliki Street, IyiAgu, Awka South, Anambra, Nigeria), snail mucin, sheep blood, Mueller-Hinton agar (Rapid Labs Colchester Essex. CO7 8SD, United Kingdom) and acetone AR (JDH GunsgdongGuandgua chemical factory Co. Ltd China), sterile distilled water (Lion water ltd, University of Nigeria, Nsukka), oil immersion.

Similarly, an African land snails (Archachatina marginata) used in for mucin extraction were purchased from Orba market, Nsukka Local Government and $24 \mathrm{~h}$ broth Culture of clinical isolates of Streptococcus pneumonia used were isolated from sputum sample of in-patient of Nsukka district hospital both in Enugu state.

\section{METHODS}

\section{Extraction of snail mucin (slime)}

The method of Adikwu and Nnamani ${ }^{23}$ was employed for the extraction with some modifications as described. ${ }^{24}$ The African land snails ( $A$. maginata) were washed with clean water and the shells were removed by breaking the apex and a metal rod was inserted to remove the fleshy body by swirled the rod clock-wise in the shell which forced the its meat part out of the shell into a bowel. The mucus layer (slime mucus) was gently scraped off from the fleshy parts by gentle press using distilled water, pooled together in a clean closed bowel and precipitated with chilled acetone. These precipitates were then dried with a flush of cold air $\left(-4^{\circ} \mathrm{C}\right)$ to obtain greyish-brown flakes of the snail mucin, which were powdered and used for the study.

\section{Preparation of media and reagent}

Mueller-Hinton agar $(38 \mathrm{~g} / \mathrm{L})$ and blood agar base $(42 \mathrm{~g} / \mathrm{L})$ used were prepared following manufacturer's specification by dispersing the required quantity the powder in distilled water, homogenized and then sterilized with autoclave at $121^{\circ} \mathrm{C}$ for $15 \mathrm{~min}$. The blood agar was enriched with $5 \%$ sheep blood after sterilization.

\section{Collection of specimen sample}

Swab sample of the sputum swabs were collected using sterile swab sticks from the hospitalized patient and soaked with normal saline with the help of Physician and Medical Laboratory scientist. The samples were transported to the lab within four (4) hr of collection in an enclosed sterile canister in a sterile normal saline for further work.

\section{Isolation and characterization of Streptococcus pneumoniae}

The clinical samples solution in normal saline were inoculated onto an enriched sterile Columbia blood agar and incubated under a reduced oxygen at $37^{\circ} \mathrm{C}$ for $24-36 \mathrm{~h}$. The resultant isolates were further purification and characterized.

\section{- Morphological characterization and identification}

The isolates were characterized and identified by surface colonial morphology, hemolytic production on 5\% sheep supplemented blood agar, ${ }^{25}$ and gram staining reaction under microscopic examination with oil immersion objective lens.

\section{- Biochemical tests confirmation}

The suspected Streptococcus specie were further subjected to biochemical tests of catalase and bile solubility. ${ }^{26}$ 


\section{Evaluation of combined activity of lincomycin and snail mucin using checkerboard}

An overnight broth culture of the test organism was use to obtain bacteria suspension of $1.5 \times 10^{8} \mathrm{cfu} / \mathrm{ml}$ in sterile normal saline in comparism with 0.5 Marcfarland standard. ${ }^{4}$ The checkerboard method of fractional inhibitory concentration (FIC) index also called activity index was adopted in order to determine the FIC index of the bacteriostatic activity of the combination of lincomycin and snails mucin (animal extract). The separate solutions of the lincomycin and mucin were prepared with sterile distilled water, each solution containing twice the MIC. The stock solution of thesnail mucin (animal extract) $50 \mathrm{mg} / \mathrm{ml}$ and stock solution of lincomycin $50 \mu \mathrm{g} / \mathrm{ml}$ were used for evaluation of their combine effect on 6 strains of Staphylococcus aureus.

The two agents were mixed in varying ratios ranging of 0:10 to 10:0 of the lincomycin and snail mucin in accordance with the checkerboard stipulation. The antibiotic and mucin extract solutions were combined in different ratio adopting the continuous variation. Each combination was then diluted two folds serially up to eleven dilution in sterile normal saline with test tubes and was seeded into the pre cooled molten blood agar in a sterile petri dish which was allowed to solidify. The six (6) strains of the test organism each was streaked on each of the surface of the agar and was incubating at $37^{\circ} \mathrm{C}$ for $24 \mathrm{hr}$. The combine effect of the antimicrobial agent on each of the test organism were determined and recorded for further analyses to draw the nature of the interaction.

\section{Analysis}

The data was analyze for standard deviation using SPSS 7.0

\section{RESULTS}

The microscopic and biochemical identification of the test S. pneumonia.

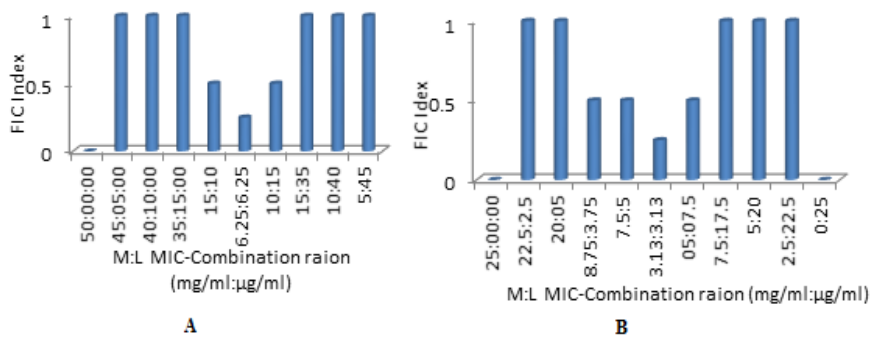

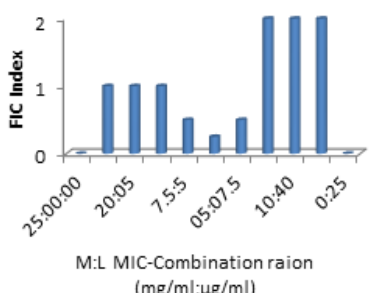

$(\mathrm{mg} / \mathrm{ml}: \mu \mathrm{g} / \mathrm{ml})$

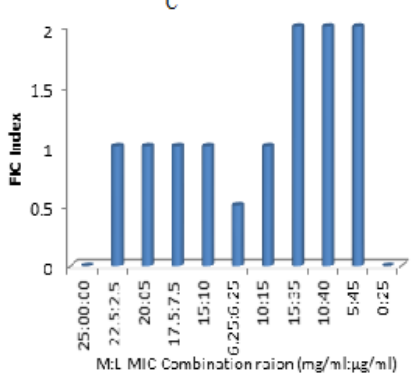

E

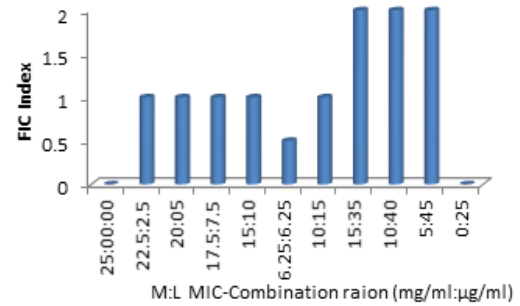

D
Figure 2: a. Effect on Nose (Sputum) isolate SN22, b. Effect on Throat (swab) isolate ST9, c. Effect on Rectal (stool) isolate SR10, d. Effect on Genital (HVS) isolate SG-HVS20, e. Effect on Genital (U/swab) isolate SG-US34, f. Effect on Skin isolate SS17.

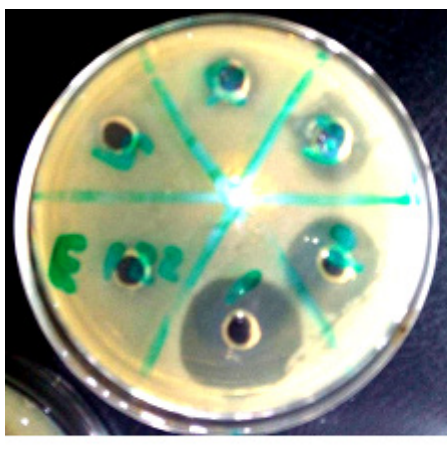

a

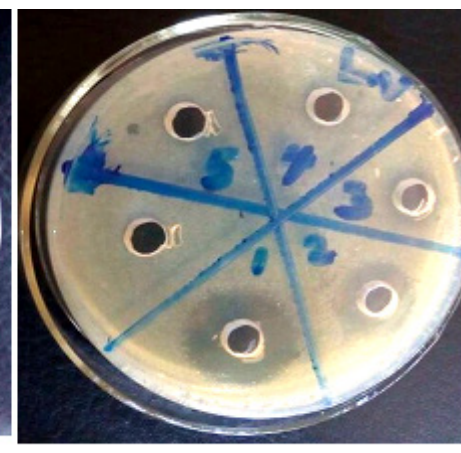

b

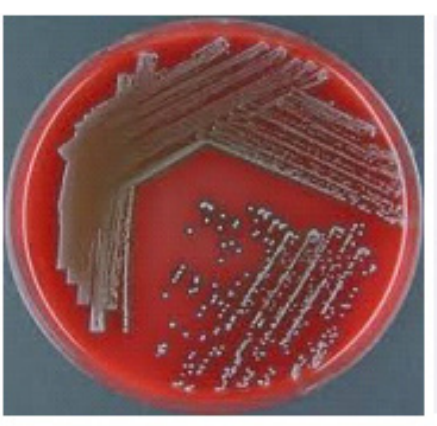

a

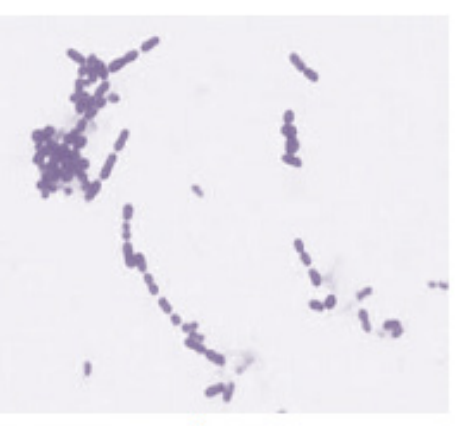

b
Figure 1: $a$ and $b$ : The colony and Gram. Morphologies.
Figure 3: a. Effect of Lincomycin against S. pneumoniae, b. effect of snail mucin against $S$. pneumonia.

\section{DISCUSSION}

Achatina fulica is a land snail capable of producing a glycoproteic secretionwhich that has been demonstrated to present some biological effects. ${ }^{27}$ Furthermore, mucus of this snail has been tested in surgical wounds of experimental animals and proven to improve the dermal cicatricial repair. ${ }^{28}$ The checkerboard assay method was used to test the activities of lincomycin and mucin in combination against strains of Streptococcus pneumoniae from different routes of the bacterial infection to human like skin, genital, rectal and throat through mouth ${ }^{29}$ 
by determining the FICs indecs of all the combinations tested. The result obtained from the research conducted above had that, the interaction of lincomycin and snail mucin against the Streptococcus pneumoniae strains used which represented with the code "S" had good effect. This is in line with the report of water soluble fraction (WSF) and mucus fraction (MF) shown positive antibacterial activity for both the Gram positive bacteria, ${ }^{14}$ Bacillus subtilis and Staphylococcus aureus, which exhibit the same physiological response with Strep. species as Gram positive bacteria to antimicrobial agents when assayed by the paper-disc method. Table 1 showed that the isolated organisms were Gram positive cocci arranged in chain by its Gram reaction and morphologies and consequently confirmed positive to catalase and bile solubility biochemical tests and its colony and structural characteristics as shown in Figure 1 a and a on the blood agar as a physiological response of alpha-hemolysis which is a product of a hemolysin that belongs to the family of sulfhydryl (SH)activated hemolysins and is commonly referred to as pneumolysin by Streptococcus pneumoniae. ${ }^{30}$ The combination of the drug and the snail mucin as a natural antimicrobial agent as shown in some recent studies showed better effect which can contribute in boosting of immune system as a result of protein content of the mucin and large carbohydrate-rich molecules. ${ }^{31}$ Figure $2 \mathrm{a}$ and $\mathrm{b}$ had similar synergistic effect at $50 \%$ (M:L 6.25:6.25 and 3.13:3.13) combination with a little difference effect at 25\% and 75\% (M:L 10:15 and 15:10) respectively while other combination ratio had the same effect of additive at FIC index of 1.0. This nose and throat has been reported as a major clinical sources of such isolate which are linked as upper respiratory system of alimentary canal which harbor similar set of infectious agents like Streptococcus pneumoniae, which initially inhabits the mucosal surfaces of the nasopharynx in its hosts. ${ }^{32,33}$ Figure $2 \mathrm{c}$ showed the FIC index less than 0.5 at the combination of M:L (3.13:3.13) and 0.5 (M:L 7.5:5.5) as a better effect of synergism when the FIC index is less than 1.0 while Figure $2 \mathrm{~d}$, e and $\mathrm{f}$ showed the combination effect of the mucin and lincomycin against $S$. pneumoniae isolates from rectum, genital organ and skin respectively with the FIC index of 0.5 and 1.0 as 50\% (M:L 6.25:6.25 and 10:15). Generally, the combinations of the two agent's sowed higher synergistic effect at the $50 \%$ each of the ratio combination against all the test isolates with the FIC index less than 0.5 . This suggest better performance of equal ration combination in concentration of the two agents to avoid side negative effect of possible toxicity increase or precipitation which endanger the patients and antagonistic effect showing FIC index of up to 2 at ratio combination of 15:35, 10:40 and 5:45 as result of concentration variant negative combination mechanism. ${ }^{34}$ Figure 1,2 showed general percentage interaction between mucin and lincomycin which had $100 \%$ steady interaction against the isolate of skin and lower pelvic region of rectum and genital. The low percentage interaction of the drugs at the nasopharynx tract could suggest the resistance from exposure and adaptation of habited microbes to inhalation and hard drug intake as reported. Fig. 3.0 Showed the inhibition and activity pattern of the Lincomycin and Mucin against the test organisms.

\section{CONCLUSION}

The natural product drug interaction has gone a long way in enhancing the effect of most ortodox antibiotics drug when combined with another by immunomodulation and potentiating effect through activation mechanism with minimal side effect. The checkerboard method adopted here reviled that the mucin and lincomycin combination improves synergistic effect at $50 \%$ ratio combination of each agent which can be considered as alternative drug combination therapy in treatment of acute pneumonia.

\section{CONFLICT OF INTEREST}

This is an original research work done and written with high level of understanding and without any conflict of interest on the course of the research

\section{ABBREVIATIONS}

IZD: Inhibition zone diameter; HVS: Higher virginal swab; FIC: Fraction inhibitory concentration; MIC: Minimum inhibitory concentration; PBS: Phosphate buffer saline; aq.: Aqueous; min: minute; g: gram; mg: milligram; dl: deciliter; ml: millitres; l: litre; w: weight; v: volume; bw: body weight.

\section{REFERENCES}

1. Catia C, Martin-Loeches I, Garcia-Vidal C, Alicia SJ, Torres A. Microbial Etiology of Pneumonia: Epidemiology, Diagnosis and Resistance Patterns. Int J Mol Sc. 2016;17(12):2120-18.

2. Xiaowei $X$, Jingan Z, Lixin $X$, Haiyan W, Yan $X$, Yingjia $S$, et al. Identification of differentially expressed proteins in the injured lung from zinc chloride smoke inhalation based on proteomics analysis. Respiratory Research. 2019;20(1):36.

3. Catia C. Cristina D, Antoni T. Multidrug Resistant Gram-Negative Bacteria in Community-Acquired Pneumonia. Critical Care. 2019;23(1):1-9.

4. Okore VC. Chemical antimicrobial agents. In: Principles of Pharmaceutical microbiology No 12 Orba Road, Nsukka: $2^{\text {nd }}$ ed. Ephrata Publishers. 2009;90-6.

5. Nina S, Pamela JY. Suppressive drug combinations and their potential to combat antibiotic resistance. J Antibiot. 2017;70(11):1033-42.

6. Cassie RB, Morales GAL, Althaus M, Wilcox MD, Jeffrey PP, John C, et al. Evolutionary conservation of the antimicrobial function of mucus: $A$ first defense against infection. Biofilms and Microb. 2018;4(1):14.

7. Itoh Y, Kamata-Sakurai M, Denda-Nagai K, Shigenori N, Makoto T, Ishii-Schrade $\mathrm{K}$, et al. Identification and expression of human epiglycanin/MUC21: A novel transmembrane mucin. Glycobiology. 2008;18(1):74-83.

8. Durrer C, Irache JM, Duchene D, Ponchel G. Mucin interactions with functionalized polystyrene latexes. J Col Inter Sci. 1995;170(2):555-61.

9. Kenechukwu FC, Ibezim EC, Attama AA, Momoh MA, Ogbonna JDN, Nnamani $\mathrm{PO}$, et al. Preliminary spectroscopic characterization of PEGylated mucin, a novel polymeric drug delivery system. Afr J Biotech. 2013;12(47):6661-71.

10. Lee S, Muller M, Rezman K, Spencer ND. Porcine gastric mucin (PGM) at the water/poly (dimethylsiloxane) (PDMS) interface: Influence of $\mathrm{pH}$ and ionic strength on its conformation, adsorption and aqueous lubrication properties. Langmuir. 2005;21(18):8344-53.

11. Adegoke AA, Adebayo T, Bukola C, Inyang UC, Aiyegoro AO, Komolafe OA. Snails as meat source. Epidemiological and nutritional perspectives. J Micro and Antim. 2009;2(1):12-8

12. Daina I, Aleksandrs J, Vita S, Vita S. Evaluation of nutrition value of roman snail's (Helix pomatia) meat obtained in latvia. Food Balt Conference Proceedings. 2014;28-31.

13. Houndonougbo MF, Chrysostome CAAM, Odoulami RC, Codjia JTC. Snail shell as an efficient mineral feedstuff for layer hens: Effects and optimum rate. Livestock Research for Rural Development. 2012;24(9):160.

14. Sanae MMI, Ttakashi A, Juichiro JM. Antibacterial activity of snail mucus mucin. Camp Biochon Phpol. 1982;72(3):571-4.

15. Czarniak P, Boddy M, Sunderland B, Hughes JD. Stability studies of lincomycin hydrochloride in aqueous solution and intravenous infusion fluids. Drug Design Development and Therapy. 2016;10:1029-34.

16. Hassan YM, Farouk HA. Description and nomenclature of Lincomycin hydrochloride: Analytical profiles of drug substances and excipients. Toronto: Acad Press. 1994;272-4.

17. Svetolik D, Jutta ML, Vincent AF. Synergistic Killing of Streptococcus pneumoniae with the Bacteriophage Lytic Enzyme Cpl-1 and Penicillin or Gentamicin Depends on the Level of Penicillin Resistance. J Anti Agents Chem. 2005;49(3):1225-8.

18. Harti AS, Estuningsih, Kusumawati HN, Siswiyanti and Setyaningtyas A. In vitro Synergistic Effects of Snail Slime and Chitosan against Staphylococcus aureus. Int J Pharm Med Biol Sci. 2016;2:137-41.

19. Keith CT, Borisy AA, Stockwell BR. Multicomponent therapeutics for networked systems. Nature Reviews Drug Disc. 2005;4(1):71-8

20. Braberg $H$, Alexander $R$, Shales $M, X u ~ J$, Franks-Skiba KE, Wu Q, et al. Quantitative analysis of triple-mutant genetic interactions. Nature Protocols. 2014;9(8):1867-81

21. Lepper MH, Dowling HF. Treatment of pneumococcic meningitis with penicillin compared with penicillin plus aureomycin: Studies including observations on an apparent antagonism between penicillin and aureomycin. AMA Archives of Internal Med. 1951;88(4):489-94. 
22. Okore VC. Antibiotic assay and tests. In: Pharmaceutical microbiology. No 9 Ani Street, OguiNew Layout, Enugu: First ed. EL 'DEMARK Publishers. 2005;190-1.

23. Adikwu MU, Nnamani PO. Some physiological and toxicological properties of snail mucin extracted from Archachatina marginata. Bio-Research. 2005;3(2):1

24. Arhewoh IM, Eraga OS, Builders FP, Ibobiri AM. Effect of mucin extraction method on some properties of metronidaziole mucoadhesive loaded patches. J of Pharmacy and Bio. 2015;12(1):1-7.

25. Ataiza CV, Marizoneide CG, Filho MR, Filho JE, Edson JMB, Rosane BF. Streptococcus pneumoniae: A study of strains isolated from cerebrospinal fluid. J de Pediatria, 2007;83(1):21-75.

26. Ruoff KL, Whilley RA, Beighton D. Streptococcus. Manual of clinical microbiology. $8^{\text {th }}$ ed. Washington: ASM Press. 2003;405-21.

27. Wagno AS, Cláudia MM, Cardoso JC, Pereira-Filho RN, Rabelo AS, Reis FP, et al Assessment of Antimicrobial Activity and Healing Potential of Mucous Secretion of Achatina fulica. Int J Morphol. 2012;30(2):365-73.
28. Martins, MF, Caetano, FAM, Sírio OJ. Avaliação do reparo de lesões de pele de coelhostratadas com secreçãomucoglicoproteica do escargot Achatina. Braz J Vet Res Anim Sci. 2003;1(1):1-11.

29. Rajiv S, Antosh S, Sugandha S. Oral Sex, Oral Health and Orogenital Infections. J Glob Infect Dis. 2010;2(1):57-62.

30. Krzysztof K, Mollby R. Production and purification of Streptococcus pneumoniae hemolysin (pneumolysin). J of Clinical Micro. 1987;25(2):222-5.

31. Smith AM, Morin MC. Biochemical differences between trail mucus and adhesive mucus from marsh periwinkle snails. Biol Bull. 2002;203(1):338-46.

32. Brooks LRK, Mias GI. Streptococcus pneumoniae's Virulence and Host Immunity: Aging, Diagnostics and Prevention. Front Immunol. 2018;9:1366.

33. Kadioglu A, Weiser JN, Paton JC, Andrew PW. The role of Streptococcus pneumoniae virulence factors in host respiratory colonization and disease. Nat Rev Microbiol. 2008;6(4):288-301.

34. Foucquier J, Guedj M. Analysis of drug combinations: Current methodological landscape. Pharmacol Res Persp. 2015;3(3):e00149.

Article History: Submission Date : 07-06-2020; Revised Date : 22-06-2020; Acceptance Date : 31-07-2020.

Cite this article: Gugu TH, Onwusoba RC, Onyi PN, Ozioko AC. Synergistic Interaction of Natural Snail Mucin and Lincomycin for Immuno-chemotherapy against Streptopneumococcal Infection: Checkerboard Evaluations. Int. J. Pharm. Investigation, 2020;10(3):379-83 